\title{
FETAL HEART SIGNAL MONITORING WITH CONFIDENCE FACTOR
}

\author{
Jianfeng Chen, Koksoon Phua, Ying Song, Louis Shue
}

Institute for Infocomm Research, 21 Heng Mui Keng Terrace, 119613, Singapore

\begin{abstract}
In this paper we present a PDA-based fetal heart monitor that is able to provide instantaneous fetal heart rate (FHR) for the pregnant women. A modified spectral subtraction algorithm is first used to remove the unwanted stationary background noise from the noisy fetal heart sound. The AM/FM modulation technique is then employed to make the fetal heart sound more audible so that both pregnant women and gynaecologists can identify the rhythmic fetal heart beat sounds easily. A real-time algorithm is proposed to extract the fetal heart rate information from the weak and noisy signals. Furthermore, based on the signal spectrum distribution, a confidence factor (CF) is designed to indicate the reliability of the estimated fetal heart rate in real time. The device has been tested in a local hospital on a sample group of 41 pregnant women. Our finding shows that an approximate $75 \%$ of the pregnant mothers are able to hear their baby's heart sound clearly using our device. Tests are also carried out to compare the fetal heart rate calculated using our device and the existing ultrasound cardiotocography (CTG) machine. In most time our device functions properly and provides results close to the CTG records.
\end{abstract}

\section{INTRODUCTION}

Fetal heart rate (FHR) monitoring provides instantaneous information about the general well-being of the fetus [1]. Traditional fetal surveillance relies heavily on the use of cardiotocography (CTG) which is a ultrasonic techniques where long term and frequent exposure to the ultrasound radiation is not recommended for both fetus and mother [2][3]. Hence, several passive techniques have been proposed to replace the ultrasonic means, namely the fetal electrocardiography (fECG), the fetal magnetic cardiography (fMCG) and the fetal phonocardiogram (fPCG). However, we note that fECG highly relies on proper placement of multiple electrodes and fMCG utilizes big and expensive magnetic sensors, which hinder them from being used as a portable device.

The PCG signals are produced by the opening and the closing of the fetal heart valves and are captured by simply placing an acoustic sensor on the mother's abdomen. Compared with the above techniques, the fPCG has two unique advantages. Being a passive system, no energy is emitted at all and it eliminates the risk of harmful exposure to both mothers and babies. Secondly, the fPCG signals can be conveniently captured by placing a small acoustic sensor on mother's abdomen without the use of gel as in the CTG monitor. The energy requirement and the size are also trivial, which in turn offers greater portability. This promises the potential of home use and facilitates portable implementation. On the other hand, there are two technical issues to be overcome before using the fPCG. Due to the nature of the fetal heart sound, the sensor must be sensitive enough to pick up the weak and low frequency $(35-110 \mathrm{~Hz})$ vibrations from the maternal abdominal surface. Secondly, the methods must be robust to calculate FHR from the fetal heart sound which are contaminated by various maternal organ sounds, the fetal movement effect, the shear noise, etc.

The majority of the early works in this area are focused on sensor development [4]-[6]. More recent studies, on the other hand, concentrate on the FHR estimation. However, many methods [7]-[11] could not deal with the noisy fetal heart sound in the presence of various nonstationary interferences, except [3] and [4] in which a series of carefully designed rules were proposed to enhance the robustness of the FHR calculation. Despite these efforts, only a handful of these techniques progressed beyond the stage of research to become a practical application instrument that is suitable for clinical or home use. Among these techniques is the prototype from NASA Langley research centre [5] and the works conducted by Technical university of Budapest. Motivated by these needs, we conducted our study specifically on the fetal heart sound audibility enhancement, the FHR calculation under real situation and the instantaneous FHR result evaluation. The work was finally implemented on a HP iPaq hx4700 and had been tested on a group of 41 pregnant women in a local hospital.

The paper is organized as follows. In Section II, we introduce the structure of the proposed system and the function of each module. The PDA implementation is described in Section III and the clinical trail methodology and results are reported in Section IV. In the end we draw our conclusions in Section V.

\section{PROPOSED SYSTEM}

The block diagram of our proposed system is shown in Figure 1. An electret microphone is used and attached to an inverse-horn-shaped cone [1]. The weak signal from the 
microphone is pre-amplified, low-pass filtered, and then digitized for subsequent processing as follows.

\section{A. Analog and Digital Preprocessing}

As reported in [2], the main components of the fetal heart sound fall into $35-110 \mathrm{~Hz}$. Hence, an 8-order low pass analog filter chip (MAX7400) working at $110 \mathrm{~Hz}$ cut-off frequency is firstly used in our analog circuit. After sampling at $2000 \mathrm{~Hz}$, the captured discrete fetal heart sound signal $x[k]$ is then filtered using an 8-order Butterworthtype high pass IIR digital filter to attenuate components below $35 \mathrm{~Hz}$.

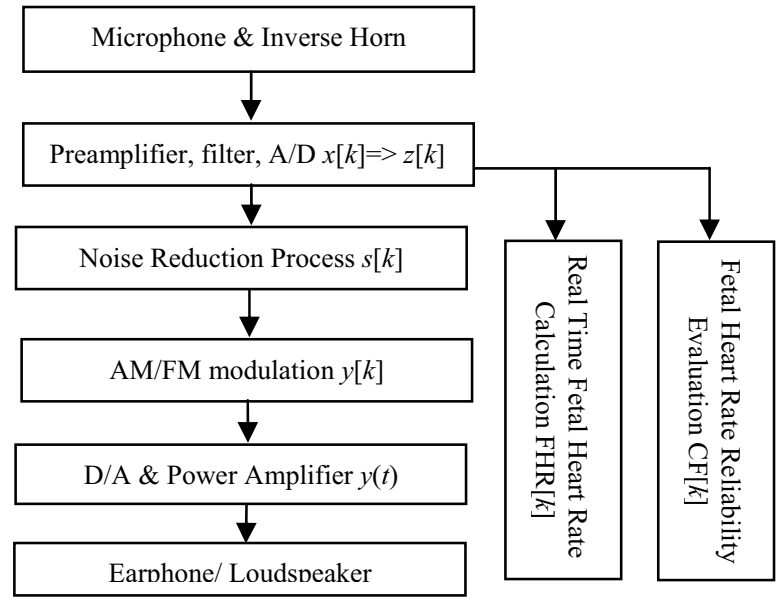

Figure 1: Block diagram of the proposed system

\section{B. Simplified Spectral Subtraction}

To further reduce the noises whose spectrum overlap with that of the fetal heart sound, we propose to use the spectral subtraction method originated from speech enhancement [12][13]. Unlike speech signal, the fetal heart sound is periodical and composed of two short pulses $\left(\mathrm{S}_{1}\right.$ and $\mathrm{S}_{2}$ ) with its large portion being noise. To explore this $a$ priori feature, we set the frame size [12] to be $30 \mathrm{~ms}$, which is less than one tenth of a normal fetal heart period (300$600 \mathrm{~ms}$ ). Hence, there must be some frames containing no fetal heart sound, which can be readily used for noise estimation. This modified spectral substraction method significantly reduces the complexity of the original method.

As mentioned earlier, the noises in the fetal heart sound include both stationary and nonstationary sources. For stationary noise, such as the slow variant organ movement noise and the electric self-noise, this modified spectral substraction method performs well. The processed signal looks much cleaner and sounds clearer as well. However, this method is not effective in removing nonstationary noises, such as some occasional burst or impulse-like noises, and the maternal heart beat. Nevertheless, it has been found that since the rhythm of the fetal heart beat is periodical and much faster than the maternal heart rate, the negative effect of these nonstationary noises on the identification of the fetal heart beat is insignificant.

\section{Amplitude Modulation (AM)}

Fetal heart sound cannot be easily identified by most people as the human ears are insensitive to the low frequencies from about $100 \mathrm{~Hz}$ downward [14]. To make the low frequency sound more audible, we implement an amplitude-modulation (AM) process which shifts the lower frequency band to a higher band while keeping the fetal heartbeat rhythm unchanged. Based on the human ear sensitivity chart in [14], when we shift a base band signal $(35-110 \mathrm{~Hz}) 250 \mathrm{~Hz}$ higher, the audibility can be regarded as being amplified by 100-10000 times in energy.

AM can be carried out by using the Single Side Band Modulation (SSB) [15]. To determine the modulation frequency, three issues are taken into consideration, which are the sampling rate, the audibility and the experience of the users. Based on our experiments and the feedbacks from many testers, an appropriate modulation frequency was found to be within the range of $150-250 \mathrm{~Hz}$.

\section{Fetal Heart Rate Calculation}

The FHR is calculated in batch mode and the segment length is three seconds. We first calculate the amplitude envelop, $A[k]$, of the original noisy signal $z[k]$ by

$$
A[k]=\sqrt{\frac{1}{L} \sum_{j=k}^{k+L-1} z^{2}[j]}
$$

where $L$ is the frame size for envelop calculation. Then, the peaks at instant $p_{i}$ are determined by using the rules

$$
\begin{array}{r}
A\left[p_{i}\right] \geq A_{\text {Thd }} \& A\left[p_{i}\right] \geq A\left[p_{i}-1\right] \& \quad A\left[p_{i}\right]>A\left[p_{i}+1\right] \\
i=1,2, \cdots, M(2)
\end{array}
$$

where $M$ is the number of the peaks in the segment under processing and $A_{\text {Thd }}$ a threshold to exclude trivial peaks. In many cases, it would be not easy to detect $S_{1}$ peaks [2]. On the contrary, in low SNR cases, many fake peaks may appear. As such, we propose to use some rules to select the potential $\mathrm{S}_{1}$ peaks.

Firstly, all peaks found based on Eq. (2) are reviewed in terms of their intervals. If there are adjacent peaks less than $30 \mathrm{~ms}$ in between, as in [2], we merge them in proportional to their respective peak amplitude.

Secondly, we select the most potential $S_{1}$ peaks based on their amplitude regularity. The instants, $p$,' satisfying the following condition is chosen as the next round candidates.

$$
A\left[p^{\prime}{ }_{i+1}\right]<\gamma \cdot A\left[p^{\prime}{ }_{i}\right], i=1,2, \cdots, N-1
$$

where $\gamma$ is the ratio of two adjacent $\mathrm{S}_{1}$ peaks and is set to be 2. $N$ is the number of selected $\mathrm{S}_{1}$ peaks.

The intervals between each two adjacent $S_{1}$ peaks are denoted as $q_{i}=p^{\prime}{ }_{i+1}-p_{i}^{\prime}$. Those instants producing abnormal intervals are further removed so that all the intervals used in the subsequent FHR calculation are within the predefined range, i.e.,

$$
\frac{60 F S}{F H R_{\max }}<q_{i}^{\prime}<\frac{60 F S}{F H R_{\min }}, i=1,2, \cdots, K
$$

where $F H R_{\min }=100 \mathrm{BPM}$ and $F H R_{\max }=200 \mathrm{BPM} . K$ is the number of the final $S_{1}$ peaks in the current segment. 
Regardless of occasional interference peaks, stronger peaks normally indicate more reliable measures. Therefore we highlight these cycles by weighting with their respective envelop amplitude $A[k]$. In the end, the weighted fetal heart beat period $T_{w}$ is obtained by

and the FHR is given by

$$
T_{w}[k]=\frac{\sum_{j=1}^{K-1}\left(A\left[p^{\prime_{j+1}}\right]+A\left[p^{\prime}{ }_{j}\right]\right) q^{\prime_{j}}}{\sum_{j=1}^{K-1}\left(A\left[p^{\prime}{ }_{j+1}\right]+A\left[p^{\prime}{ }_{j}\right]\right)}
$$

$$
F H R[k]=\frac{1}{T_{w}[k]}
$$

\section{E. Confidence Factor}

Due to the highly non-stationary characteristics of the fetal heart sound itself and the unforeseeable noise condition, the calculated FHR in some time may be more reliable than others. Through observing large amount of data captured in our clinical trial and their corresponding FHR results, we notice that the fetal heart sound needs to have a high SNR ratio with a series of regular and distinct peaks of $\mathrm{A}[\mathrm{k}]$ to guarantee a reliable FHR result. Through our clinical trial it was revealed that the FHR accuracy highly relies on the regularity of the fetal heart signals. Hence we propose to quantify the signal regularity based on the spectrum distribution.

The method is derived from the spectrum characteristics of the fetal heart signals with various qualities. It has been observed that if the fetal heart sounds are regular, (1) there is usually a dominant and highest peak within the range 1.67-3.33 Hz, corresponding to $100-200$ beat per minute, and (2) the components below $1.67 \mathrm{~Hz}$ are much weaker and beyond $3.33 \mathrm{~Hz}$ some strong peaks at the FHR harmonic frequencies may appear (see Fig. 2).

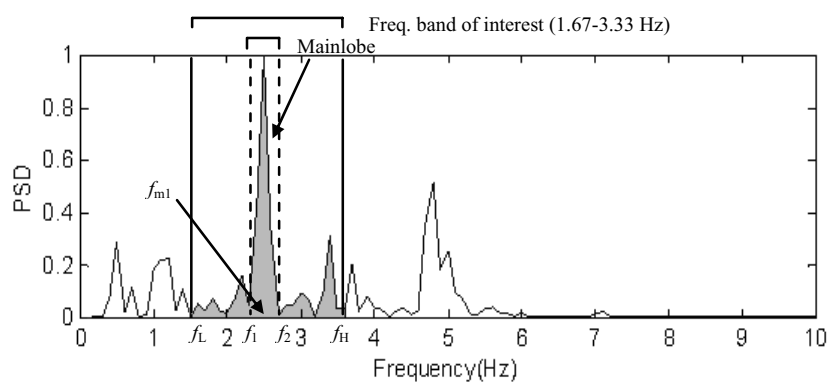

Fig. 2: Spectrum of a segment of fetal heart sound signal at $0-10 \mathrm{~Hz}$. The interested bandwidth is highlighted using shading colour and the main lobe of the dominant peak inside is marked using two dashed line

Based on these observations, we define the fetal heart rate confidence factor $(\mathrm{CF})$ as

$$
C F=\frac{E_{m}}{E} \cdot \frac{E_{A}\left(f_{m 1}\right)}{E_{A}\left(f_{m 2}\right)}
$$

where $E$ and $E_{\mathrm{m}}$ are the energy (area) of the frequency band 1.67-3.33 Hz and that of the main lobe (see Fig. 2), respectively. $E_{\mathrm{A}}(f)$ is the energy (amplitude) at frequency $f$. $f_{\mathrm{m} 1}$ and $f_{\mathrm{m} 2}$ denote the frequencies corresponding to the maximum peaks in $1.67-3.33 \mathrm{~Hz}$ and $0-3.33 \mathrm{~Hz}$, respectively.

We can see that the $\mathrm{CF}$ ranges from 0 (least reliable) to 1 (most reliable). Its first term in Eq. (6) stands for the distinctiveness of the dominant peak in $1.67-3.33 \mathrm{~Hz}$ and the second takes the negative effect of the peaks below $1.67 \mathrm{~Hz}$ into consideration.

\section{PROTOTYPE IMPLEMENTATION}

The real-time system was implemented using HP iPaq hx4700 as shown in Figure 3. On the left is the Teflonmade inverse horn with a Panasonic omni-directional microphone embedded in its bottom. The preamplifier, battery and low pass filter circuit board are all enclosed in the black box. The data acquisition unit, digital signal processing and display are performed on the iPaq. The iPaq has an Intel XScale $624 \mathrm{MHz}$ processor, $128 \mathrm{MB}$ Flash ROM, 64 MB SDRAM, 4',VGA display and a 16 bit sound processor with an audio jack for input and output.
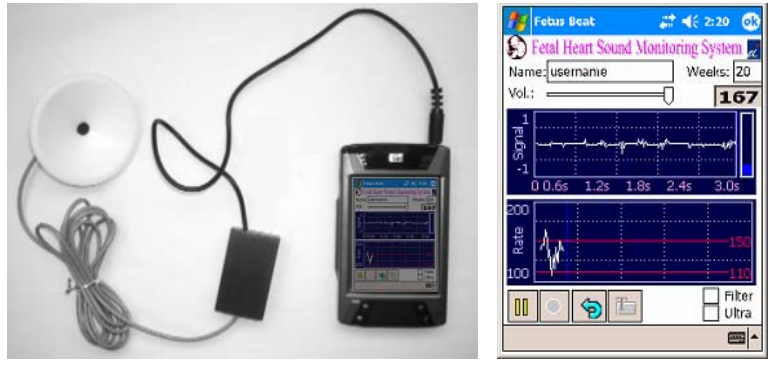

Figure 3: A portable fetal heart monitor device using a HP iPaq hx4700, a white Teflon-made cone, a Panasonic omni-directional microphone and the preamplifier and filter analog circuit.

The user interface was developed using the Embedded $\mathrm{VC}++4.0$ on the Pocket PC 2003 OS platform. The system can capture the signal from the sensors and display them on the LCD screen in real time. At the same time, all the signal processing modules are running behind the interface. The calculated FHR trace is shown below the signal waveform, as seen in Figure 3. The user can hear the processed, clean fetal heart sound through an earphone (not shown in the figure). The confidence factor is displayed in different colours in the FHR trace, indicating the reliability of the current FHR result. The whole device is light, compact and easy to use.

\section{CLINICAL TRIAL RESULTS}

Clinical trials were carried out in a local hospital on 41 pregnant women who were about to give birth (37-38 weeks). Our sensor was placed just beside the CTG sensors and for each trial ten minutes data from both devices were recorded simultaneously for subsequent comparison.

The clearness of the fetal heart sound is an important usability indicator for layman users. To quantitatively evaluate the performance of our device, the processed signals are classified into 5 grades. A subjective listening test was carried out by the pregnant women and our staff. The resulted clearness grade of those 41 fetal heart sound signals is shown in Table I. From Table 1 we observe that 
$75 \%$ of the fetal heart sound has a clearness level of 3 and above, indicating that most of these signals are clear and distinct. In contrast, it was shown during our clinical trial that few pregnant women could identify their original fetal heart beat by listening to the unprocessed signals.

\begin{tabular}{|l|c|c|}
\hline \multicolumn{1}{|c|}{ Clearness Grade } & Number & Percentage \\
\hline 5: Very distinct \& loud & 17 & $41 \%$ \\
\hline 4: Distinct and loud & 7 & $17 \%$ \\
\hline 3: Clear & 7 & $17 \%$ \\
\hline 2: Need to listen attentively & 6 & $15 \%$ \\
\hline 1: Cannot hear at all & 4 & $10 \%$ \\
\hline Total & 41 & $100 \%$ \\
\hline
\end{tabular}

TABLE I: CLEARNESS RESULT OF CLINICAL TRIAL
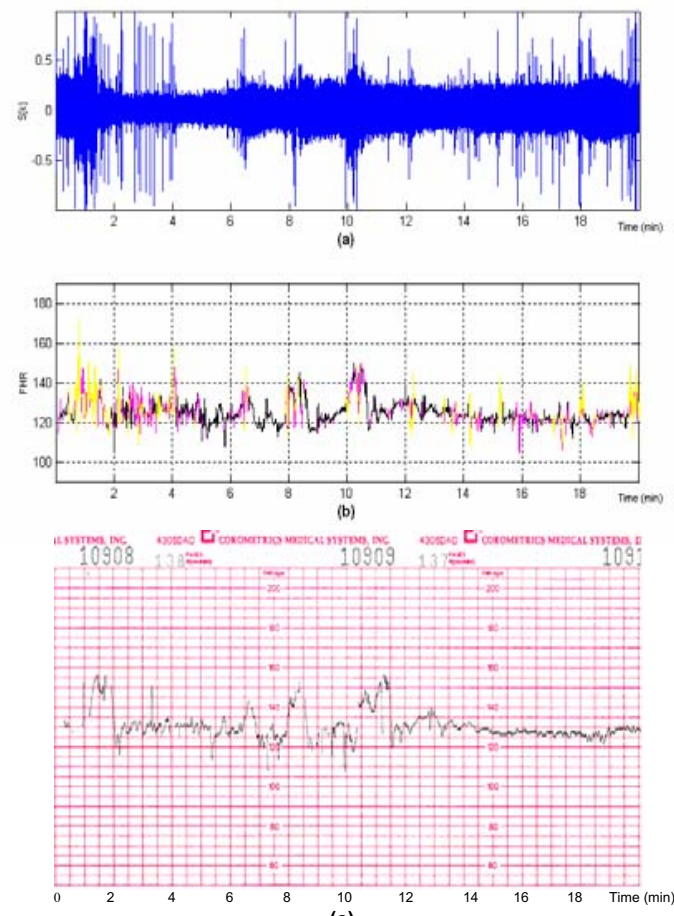

(c)

Figure 4: Comparison of FHR traces obtained by our device and CTG machine. (a) 20 minutes original fetal heart sound data; (b) FHR trace obtained by our device; (c) FHR trace obtained by CTG machine. Note: the Confidence Factor is also demonstrated in (b) by different colours, Black: $\mathrm{CF}>0.7$; Pink: $0.35<\mathrm{CF}<0.7$; Yellow: $\mathrm{CF}<0.35$.

The experiments on synchronized comparison between Sonicaid Fetal CTG machine and our device have also been carried out. It is found that it would be less easy than CTG to get a long term stationary signal with distinct fetal heart beat as the mother may move around, talk, or touch the quilt, etc, which affects the continuity of our device. A typical result is shown in Figure 4 to give a representation of how effective our system may work in normal conditions. It can be seen that aided with the confidence factor derived above, as highlighted in the figure using different colours, the users can perceive the reliability of the FHR results objectively. In most time, when the CF is high, our device functions well and provides results very close to the CTG records.

\section{CONCLUSION}

A portable fetal heart sound monitor has been developed on a HP iPaq hx4700. The processed fetal heart sound can be easily identified by layman users. Assisted with the confidence factor, the reliability of the obtained FHR can be intuitively evaluated. The confidence factor can in turn be used as an indicator to search the optimal fetal heart sound auscultation location. On the other hand, we realize that the PPCG performance is not as good when compared to the CTG, especially in high interference situations. Therefore we suggest using the device as a supplementary tool rather than a substitution for the ultrasound device. It can also be used as a prescription device at home or in office, especially during the last few weeks of pregnancy or for high risk pregnant women.

\section{REFERENCES}

[1] R. K. Freeman, T. J. Garite, Fetal Heart Rate Monitoring, chapter The Physiologic Basis of Fetal Monitoring, Williams and Wilkins, Baltimore, Maryland, 1981, pp. 7-17

[2] P. Várady, L. Wildt, Z. Benyó, A. Hein, An advanced method in fetal phonocardiography, Computer Methods and programs in Biomedicine, 71, 2003, pp. 283-296

[3] F. Kovács, M. Török, I. Habermajer, A rule-based phonocardiographic method for long-term fetal heart rate monitoring, IEEE Trans. Biomed. Eng., 47, 2000, pp. 124-130

[4] D.G. Talbert, W. L. Davies, F. Johnson, N. Abraham, N. Colley, D. P. Southall, Wide bandwidth fetal phonography using a sensor matched to the compliance of the mother's abdominal wall, IEEE Trans. Biomed. Eng. vol. 33, no. 2, 1986, pp. 175-181

[5] A. J. Zuckerwar, R.A. Pretlow, J. W. Stoughton, et al, Development of a piezopolymer pressure sensor for a fetal heart rate monitor, IEEE Trans. Biomed. Eng., vol. 40, no. 9, 1993, pp. 963-969

[6] H. G. Goovaerts, D. Cohen, J. H. Dripps, R. Mantel, P. Mooney, H. W. Jongsma, K. J. Dalton and H. P. van Geijn, A comparative clinical study of fetal phono- and movement-sensors from Amsterdam, Cambridge and Edinburgh, Journal of Biomed. Eng., vol. 13, 1991, pp. 495-499

[7] M. Godinez, A. Jimenez, M. R. Ortiz, M. Peňa, On-line fetal heart rate monitor by phonocardiography, Proceedings of the 25th Annual International Conference of the IEEE Engineering in Medicine and Biology Society, vol. 4, 17-21 Sept. 2003, pp.3141 - 3144

[8] A. Jiménez, M. R. Ortiz, M. A. Peňa, S. Charleston, R. González, A. T. Aljama, S. Carrasco, Performance of a method to generate fetal cardiotachograms using fetal phonocardiography, Computers in Cardiology, 28, 2001, pp. 453-456

[9] B. H. Tan, M. Moghavvemi, Real time analysis of fetal phonocardiography, Proc. TENCON, vol. 2, 2000, pp. 135-140

[10] F. Kovács, M. Török, An improved phonocardiographic method for fetal heart rate monitoring, Proceedings of the 20th Annual International Conference of the IEEE Engineering in Medicine and Biology Society, vol. 20, No. 4, 1998, pp. 1719-1722

[11] M. A. Peňa, R. Gonzalez, A. T. Aljama, S. Carrasco, R. Ortiz, C. G. Vargas, G. Valencia, Comparison of abdominal ECG and phonocardiography for instantaneous fetal heart rate detection, Proc. IEEE-EMBC and CMBEC, 1997, pp. 123-124

[12] Y. Ephram, D. Malah, Speech enhancement using a minimum meansquare error short time spectral amplitude estimator, IEEE Trans. On acoustics, speech, and signal processing, vol. ASSP-32, no. 6, Dec. 1984, pp. 1109-1121

[13] Rainer Martin, Noise power spectral density estimation based on optimal smoothing and minimum statistics, IEEE Trans. On Speech and Audio Processing, vol. 9, no. 5, 2001, pp. 504-512

[14] Davis, H., \& Silverman, S. R. Hearing and Deafness, Holt, Rhinchart and Winston, New York, 1978

[15] B.P. Lathi, Modern Digital and Analog Communications Systems, 3rd Edition, OUP, 1998 